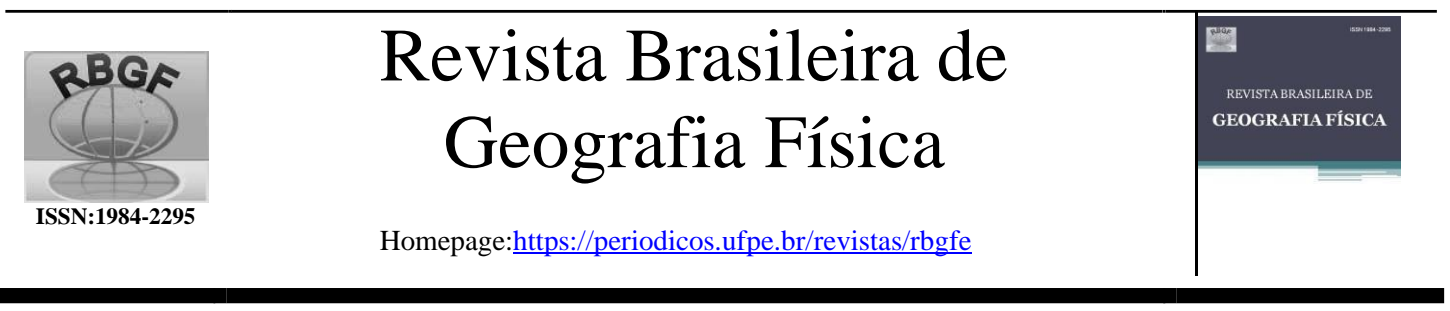

\title{
Climatologia da Chuva em Maceió: Aspectos Climáticos e Ambientais
}

José Francisco de Oliveira Júnior ${ }^{1}$, Pedro Henrique de Almeida Souza ${ }^{1}$, Edson de Oliveira Souza ${ }^{1}$, Mário Henrique Guilherme dos Santos Vanderlei ${ }^{1}$, Washington Luiz Félix Correia Filho ${ }^{1}$, Carla Taciane Brasil dos Santos ${ }^{1}$, Bárbara Alves Batista ${ }^{1}$, Dimas de Barros Santiago ${ }^{2}$, Givanildo de Gois ${ }^{3}$

${ }^{1}$ Laboratório de Meteorologia Aplicada e Meio Ambiente - LAMMA, Instituto de Ciências Atmosféricas (ICAT), Universidade Federal de Alagoas (UFAL), Maceió, Alagoas. jose.junior@icat.ufal.br/ pedro.meteorologia@gmail.com/edson_eng_agri@yahoo.com.br/marioguih@gmail.com/wlfcfm@gmail.com/carlataciane01@gmail.com/barbara.alb a18@gmail.com; ${ }^{2}$ Universidade Federal de Campina Grande (UFAL), Campina Grande, Paraíba. dimas.barros91@gmail.com; ${ }^{3}$ Fundação Universidade Federal De Rondônia - UNIR, Porto Velho, Rondônia. givanildogois@ gmail.com.

Artigo recebido em 23/03/2021 e aceito em 16/06/2021

\section{R E S U M O}

Os objetivos do estudo são: i) avaliar a climatologia da chuva em Maceió baseado em dados observados, com ênfase em aspectos climáticos e ambientais e ii) validar o produto de precipitação para município. Dados de 1979 a 2013 do produto de precipitação CHELSA (Climatologies at High Resolution for the Earth's Land Surface Areas) foram validados pelos dados pluviométricos oriundos da Agência Nacional de Águas (ANA) no período de 1960 a 2016 . Os indicadores estatísticos mostraram um alto coeficiente de determinação e correlação linear entre dados CHELSA e observado $\left(\mathrm{R}^{2}=0,80 ; \mathrm{r}=0,89\right)$ e os menores erros $(\mathrm{EPE}=6,58 \mathrm{~mm}$ e RMSE $=18,76 \mathrm{~mm})$, portanto o produto CHELSA pode ser aplicado na região. A série temporal apresentou um período 1 (P1) - (1960 a 1989) com chuvas superiores à média histórica e um período 2 (P2) - (1990 a 2016) com redução significativa da chuva. Dados observados versus as normais climatológicas mostrou uma diminuição significativa na normal 1 (1961-1990) na estação chuvosa, enquanto em relação a normal 2 (1981-2010) houve aumento nos meses de fevereiro, março e abril (entre 10 a $20 \%$ ) e outubro e dezembro (entre 5 a 15\%). A distribuição espacial da chuva mensal via produto CHELSA mostrou a formação de um gradiente pluviométrico entre a costa e parte alta de Maceió. A topografia influencia o regime de chuva nos bairros pertencentes às regiões administrativas - RA (R4, R5 e R6) com maiores registros de chuva. As fases do ENOS são responsáveis diretos na variabilidade da chuva interanual, enquanto a variabilidade decadal correspondeu à mudança de fase da ODP e as alterações no uso e ocupação da terra em Maceió.

Palavras-chave: CHELSA, chuva urbana, urbanização, adensamento populacional, vulnerabilidade socioambiental.

\section{Climatology of Rainfall in Maceió: Climatic and Environmental Aspects}

\section{ABSTRACT:}

The objectives of the study are: i) to evaluate the climatology of rain in Maceió based on observed data, with emphasis on climatic and environmental aspects and ii) to validate the precipitation product for the municipality. Data from 1979 to 2013 of the precipitation product CHELSA (Climatologies at High Resolution for the Earth's Land Surface Areas) were validated by rainfall data from the National Water Agency (NWA) from 1960 to 2016. Statistical indicators showed a high coefficient of determination and linear correlation between CHELSA and observed data $\left(R^{2}=0.80 ; r=\right.$ 0.89 ) and the smallest errors ( $\mathrm{SEE}=6.58 \mathrm{~mm}$ and RMSE $=18.76 \mathrm{~mm}$ ), therefore the CHELSA product can be applied in the region. The time series presented a period 1 (P1) - (1960 to 1989) with rainfall above the historical average and a period 2 (P2) - (1990 to 2016) with a significant reduction in rainfall. Observed data versus climatological normals showed a significant decrease in normal 1 (1961-1990) in the rainy season, while in relation to normal 2 (1981-2010) there was an increase in the months of February, March and April (between 10 to 20\%) and October and December (between 5 to 15\%). The spatial distribution of monthly rainfall via the CHELSA product showed the formation of a pluviometric gradient between the coast and the upper part of Maceió. The topography influences the rainfall regime in neighborhoods belonging to the administrative regions (AR) - (R4, R5 and R6) with the highest rainfall records. The

Júnior., J., F., O., Souza., P., H., A., Souza., E., O., Vanderlei., M., H., G., S., Filho., W., L., F., C., Santos., C., T., B., Batista., B., A., Santiago., D., B., Gois., G. 
ENOS phases are directly responsible for the variability of interannual rain, while the decadal variability corresponded to the PDO phase change and changes in land use and occupation in Maceió.

Keywords: CHELSA, urban rainfall, urbanization, population density, socio-environmental vulnerability.

\section{Introdução}

O desenvolvimento econômico e sustentável de qualquer região depende de diversos fatores, principalmente da água, como fonte de vida, no abastecimento e na hidroeletricidade (Boretti e Rosa, 2019; IBGE, 2020). Assim, a compreensão da distribuição espaço-temporal da chuva é fundamente para fins climatológicos, gestão dos recursos hídricos e na segurança alimentar (FAO et al., 2016; Armstrong et al., 2020). É sabido que a dinâmica da chuva interfere no cotidiano da população e nas diversas atividades socioeconômicas (IPCC, 2018).

Nas últimas décadas, os extremos de chuva em ambientes urbanos têm sido frequentes e trazem prejuízos econômicos e mortes (IPCC, 2018; Nascimento et al., 2018; Oliveira Júnior et al., 2021). A relação entre a chuva e a sua variabilidade espaço-temporal têm sido amplamente estudadas em grandes centros urbanos (Sobral et al., 2020; Lima et al., 2021) e nas megacidades (Baklanov et al., 2016).Os problemas de expansão e ocupação desordenada do espaço urbano são fatores que contribuem para o aumento da vulnerabilidade social nas cidades, principalmente no Nordeste do Brasil (NEB) (Marengo et al., 2018; Correia et al., 2019a).

A falta de uma rede telepluviométrica em diversos municípios do Brasil, principalmente aqueles com população superior a 1.000 .000 habitantes é notória, exceto o município do Rio de Janeiro que já dispõe do Sistema Alerta-RJ desde 1997, que monitora a chuva urbana (Terassi et al., 2020). Assim, os gestores públicos falham na implantação de sistemas que possam fornecer a representatividade espaço-temporal da chuva e na disponibilidade de dados em tempo real (Santos et al., 2019). Esses dados podem auxiliar no

\section{Material e métodos}

\section{Área de Estudo}

O município de Maceió possui uma área de 503,07 $\mathrm{km}^{2}$, sendo 193,34 $\mathrm{km}^{2}$ (urbana), $285,47 \mathrm{~km}^{2}$ (rural) e $23,26 \mathrm{~km}^{2}$ (lagunar) - (IBGE, 2020). As altitudes variam de 10 a $100 \mathrm{~m}$ (Figura 1). A chuva anual média varia entre 1.400 $\mathrm{mm}$ a $1.500 \mathrm{~mm}$, sendo concentrados de abril a monitoramento de estiagens prolongadas que nas últimas décadas tem causado crises hídricas frequentes e nas chuvas extremas que dão origem as enchentes, as inundações repentinas, os deslizamentos de terra (Marengo et al., 2018; Terassi et al., 2020), seguido do aumento das doenças de veiculação hídrica, principalmente a dengue e a leptospirose (Oliveira Júnior et al., 2019).

Assim, o uso de produtos de precipitação tem se mostrado uma solução aos dados ausentes e as falhas oriundas das estações meteorológicas convencionais (EMC) e automáticas (EMAS) em diversos estudos de Climatologia, Meteorologia e Hidrologia, por exemplo, os estudos baseados na caracterização (Correia Filho et al., 2019; Sobral et al., 2020), na variabilidade, na validação (Paredes-Trejo et al., 2017; Costa et al., 2019) e na avaliação espaço-tempo da precipitação (Oliveira Júnior et al., 2021).

Atualmente, o município de Maceió já tem uma população estimada de 1.025.360 habitantes, distribuída em 50 bairros (PMASMACEIÓ, 2014; IBGE, 2020). Estudos recentes foram realizados baseados em aspectos geoambientais (Nascimento et al., 2018), impactos socioambientais (Oliveira Júnior et al., 2021) e, recentemente sobre cenários futuros de precipitação (Oliveira et al., 2021). No entanto, o município não dispõe na literatura científica de um estudo climatológico da chuva baseado em uma série histórica e da validação de um produto de precipitação. Portanto, os objetivos do estudo são: i) avaliar a climatologia da chuva em Maceió baseado em dados observados, com ênfase em aspectos climáticos e ambientais e ii) validar o produto de precipitação para município.

julho. A temperatura média de $24,2^{\circ} \mathrm{C}$ (estação seca) e entre $22,4^{\circ} \mathrm{C}$ e $25,5^{\circ} \mathrm{C}$ (estação chuvosa), respectivamente (Nascimento et al., 2018). O Plano Diretor da Cidade de Maceió vigente mostra que a área urbana consta de 50 bairros inseridos em oito regiões administrativas (RA), conforme a Figura 2 e a Tabela 1 (PMASMACEIÓ, 2014). 


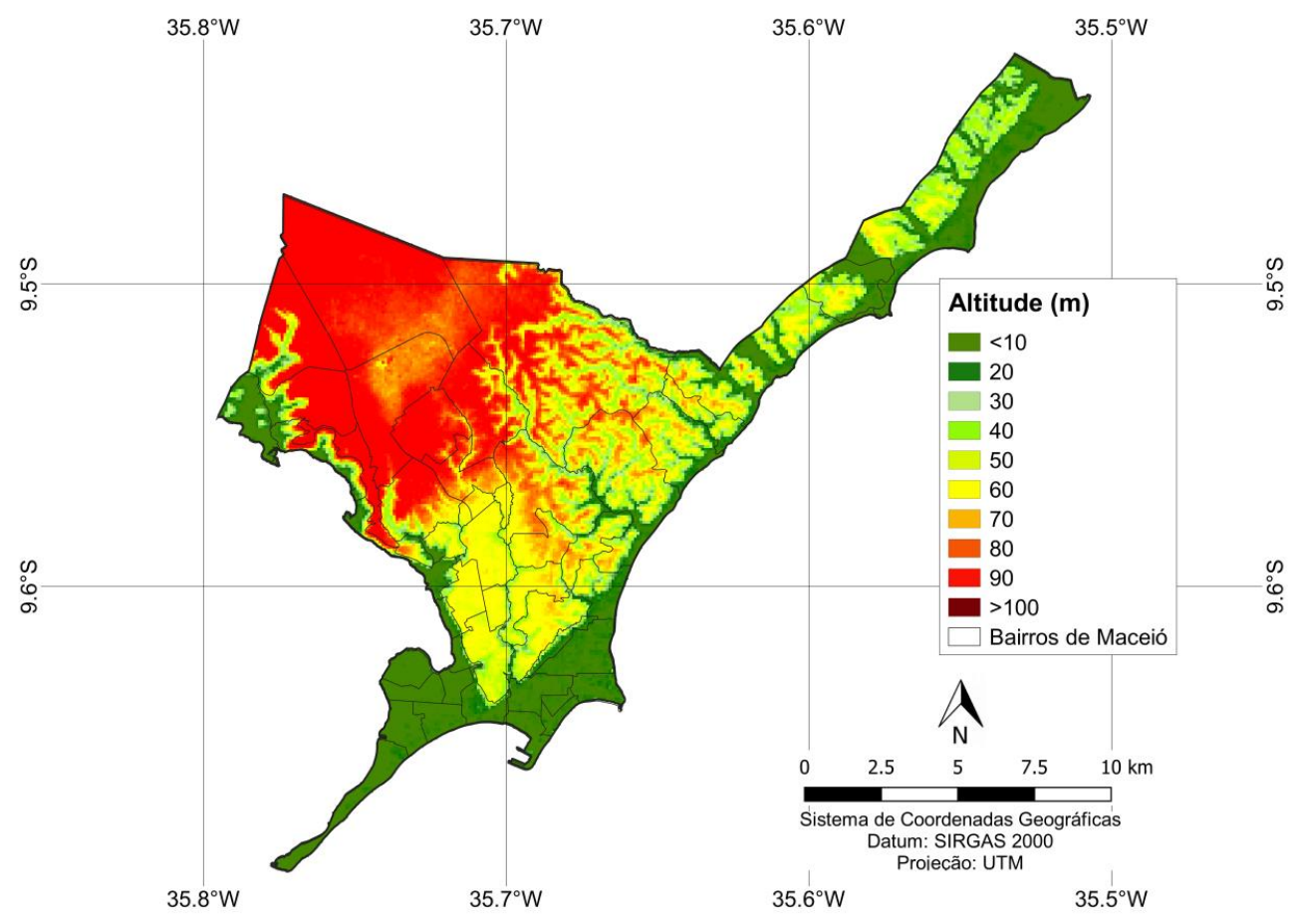

Figura 1. Mapa do munícipio de Maceió com sua altitude (m) via modelo digital de elevação (MDE).

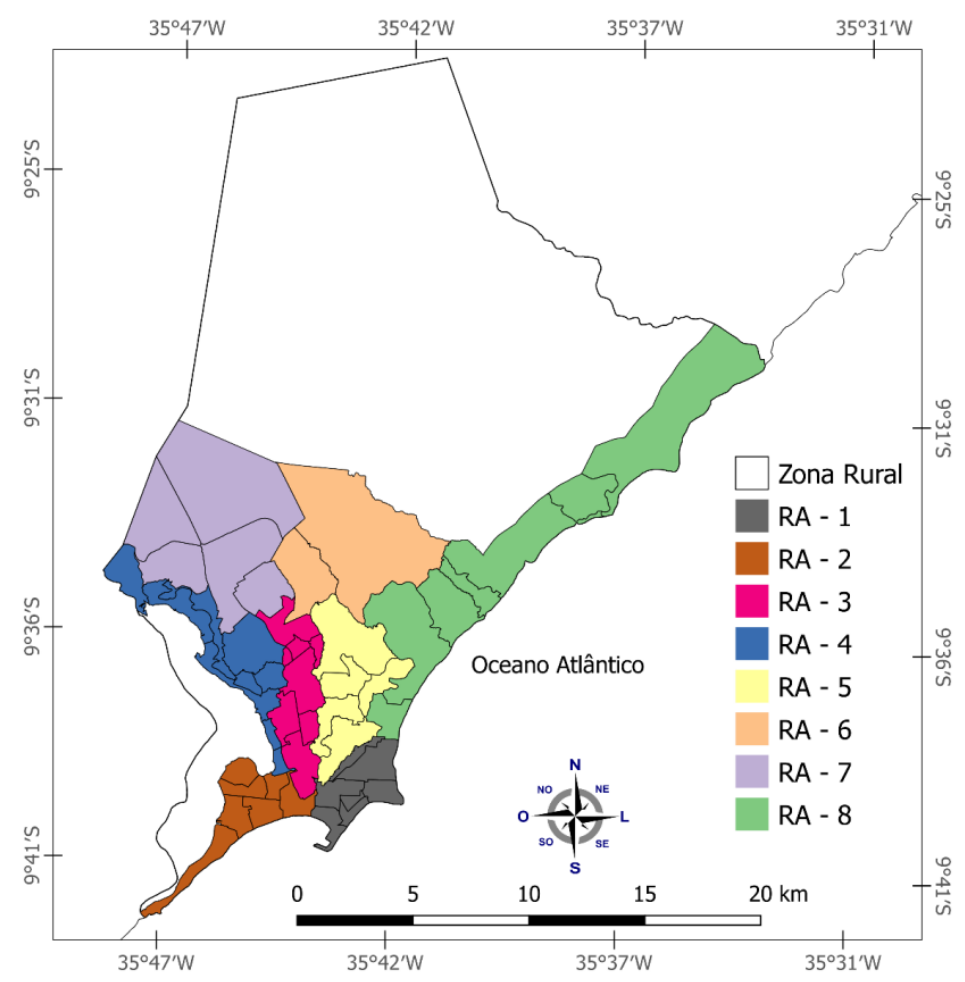

Figura 2. Mapa do munícipio de Maceió com suas 8 regiões administrativas e Zona Rural.

Júnior., J., F., O., Souza., P., H., A., Souza., E., O., Vanderlei., M., H., G., S., Filho., W., L., F., C., Santos., C., T., B., Batista., B., A., Santiago., D., B., Gois., G. 
Tabela 1. Lista dos Bairros por Região Administrativa no município de Maceió.

\begin{tabular}{|c|c|}
\hline Região Administrativa (RA) & Bairros \\
\hline RA1 & $\begin{array}{l}\text { Poço, Jaraguá, Ponta da Terra, Pajuçara, Ponta Verde, Jatiúca } \\
\text { e Mangabeiras. }\end{array}$ \\
\hline RA2 & $\begin{array}{l}\text { Centro, Pontal da Barra, Trapiche da Barra, Prado, Levada, } \\
\text { Vergel do Lago, Ponta Grossa. }\end{array}$ \\
\hline $\mathbf{R A 3}$ & $\begin{array}{l}\text { Farol, Pitanguinha, Pinheiro, Gruta de Lourdes, Canaã, Santo } \\
\text { Amaro, Jardim Petropólis, Ouro Preto. }\end{array}$ \\
\hline RA4 & $\begin{array}{l}\text { Bom Parto, Mutange, Bebedouro, Chã de Bebedouro, } \\
\text { Petropólis, Chã da Jaqueira, Santa Amélia, Fernão Velho e } \\
\text { Rio Novo. }\end{array}$ \\
\hline RA5 & Jacintinho, Barro Duro, Serraria, São Jorge e Feitosa. \\
\hline RA6 & Benedito Bentes e Antares. \\
\hline RA7 & $\begin{array}{l}\text { Santos Dumont, Cidade Universitária, Santa Lúcia, Tabuleiro } \\
\text { dos Martins e Clima Bom. }\end{array}$ \\
\hline RA8 & $\begin{array}{l}\text { Cruz das Almas, Jacarecica, Guaxuma, Garça Torta, Riacho } \\
\text { Doce, Pescaria e Ipioca. }\end{array}$ \\
\hline
\end{tabular}

\section{Dados Pluviométricos}

Os dados pluviométricos mensais utilizados no estudo correspondem à série pluviométrica histórica do período de 1960 a 2016, fornecidos pela Agência Nacional de Águas (ANA), disponíveis na plataforma Hidroweb (https://www.snirh.gov.br/hidroweb). A base de dados apresentava falhas na série temporal e, foi devidamente preenchida via método de imputação no ambiente R. Os detalhes no preenchimento das falhas via método de imputação encontra-se em Souza et al. (2021). Os dados pluviométricos mensais foram confrontados com as normais climatológicas obtidas do Instituto Nacional de Meteorologia (INMET) nos períodos de 19611990 (normal 1) e 1981-2010 (normal 2), com intuito de verificar aumento ou diminuição da chuva no munícipio de Maceió.

\section{Dados de Precipitação - CHELSA}

Para validação do produto de precipitação e avaliação espacial da chuva no munícipio de Maceió foi utilizado o produto Climatologies at High resolution for the Earth's Land Surface Areas (CHELSA) (Karger et al., 2017).

Os dados do CHELSA possuem resolução espacial de $1 \mathrm{~km}$ x $1 \mathrm{~km}$, na escala mensal, sendo usado anteriormente no NEB (Correia Filho et al., 2019b). O período de estudo corresponde aos anos de 1979 a 2013. O produto CHELSA é constituído por dados de estações meteorológicas oriundas do Global Precipitation Climate Centre (GPCC), com resolução temporal de $50 \mathrm{~km} \mathrm{x} 50 \mathrm{~km}$. Tal conjunto de dados é submetido à técnica de downscaling estatístico para o refinamento de grade de $50 \mathrm{~km}$ para $25 \mathrm{~km}$, a partir do método de interpolação B-Spline, com o auxílio dos dados de precipitação do Era-Interim.

Além disso, este produto também inclui os efeitos da topografia, do regime de vento e da altura da Camada Limite Atmosférica (CLA), ao qual há o refinamento da grade de $25 \mathrm{~km}$ para 1 $\mathrm{km}$ (Karger et al., 2017), disponíveis pelo site: $<$ http://chelsa-climate.org/timeseries/>. Eles são armazenados em arquivos de extensão do tipo Geotiff. Após, a obtenção destes arquivos, foi realizado o pré-processamento dos dados pluviométricos relativos às regiões limítrofes do munícipio de Maceió.

\section{Estatística Aplicada e as Fases do ENOS}

$\mathrm{Na}$ avaliação do desempenho dos dados do CHELSA foram usados os seguintes parâmetros estatísticos: os coeficientes de determinação $\left(R^{2}\right)$ e correlação linear de Pearson $(r)$, Erro Padrão da Estimativa $(E P E, \mathrm{~mm})$ e Raiz Quadrada do Erro Quadrático Médio (RMSE, $\mathrm{mm})$, conforme as equações a seguir. 


$$
\begin{gathered}
\mathrm{R}^{2}=1-\frac{\sum_{\mathrm{i}=1}^{\mathrm{n}}\left(\left|\mathrm{X}_{\mathrm{i}}-\overline{\mathrm{X}}_{\mathrm{o}}\right|\right)^{2}}{\sum_{i=1}^{n}\left(\left|\mathrm{X}_{\mathrm{o}}-\overline{\mathrm{X}}_{\mathrm{o}}\right|\right)} \\
\mathrm{r}=\frac{\sum_{i=1}^{n}\left(\mathrm{X}_{\mathrm{o}}-\overline{\mathrm{X}}_{\mathrm{o}}\right)\left(X_{i}-\bar{X}_{i}\right)}{\sqrt{\sum_{i=1}^{n}\left(\mathrm{X}_{\mathrm{o}}-\overline{\mathrm{X}}_{\mathrm{o}}\right)^{2}-\sum_{i=1}^{n}\left(\mathrm{X}_{\mathrm{i}}-\overline{\mathrm{X}}_{\mathrm{i}}\right)^{2}}} \\
\mathrm{EPE}=\sqrt{\frac{\sum_{\frac{i=1}{\mathrm{n}}\left(\mathrm{X}_{\mathrm{o}}-X_{\mathrm{i}}\right)^{2}}^{n-2}}{\mathrm{RMSE}^{\sum_{i=1}^{\mathrm{n}}\left(\mathrm{X}_{\mathrm{o}}-X_{\mathrm{i}}\right)}}}
\end{gathered}
$$

Em que: $n=$ número de observação; $X_{i}$ é o valor iésimo da chuva CHELSA e $X_{0}$ é o valor i-ésimo da chuva observada.

Em relação à intensidade das fases do $\mathrm{El}$ Niño-Oscilação Sul (ENOS) - (El Niño, La Niña e Neutro), segue a classificação do Oceanic Niño Index (ONI), caracterizada pela região do El Niño 3.4, e foram obtidos no National Oceanic and Atmospheric Administration/Climate Prediction Center - NOAA/CPC - (NOAA/CPC, 2020). Vale ressaltar que o uso do índice ONI foi anteriormente aplicado em estudos climatológicos para o Estado de Alagoas, com resultados satisfatórios (Lyra et al., 2017; Costa et al., 2020).

\section{Resultados e discussão}

\section{Variabilidade Anual e Decadal da Chuva}

A Figura 3 exibe a distribuição temporal da chuva e suas variações anuais no munícipio de Maceió. O munícipio de Maceió, entre 1960 a 1989 (período 1 - P1), houve diversos anos chuvosos, sendo superiores a média histórica do período $(1838 \mathrm{~mm})$, com destaque para os anos de 1964 (2455,70 mm), 1969 (2680,90 mm), 1977 $(2664,80 \mathrm{~mm})$ e $1989(2985,65 \mathrm{~mm})$. Ambos os anos citados correspondem aos eventos de ENOS (El Niño e La Niña), comuns no Estado de Alagoas, particularmente na mesorregião do Leste Alagoano (Lyra et al., 2017; Costa et al., 2020), e no NEB (Marengo et al., 2018; Correia Filho et al., 2019). Situação contrária, entre 1990 a 2016 (período 2 - P2), onde houve uma diminuição significava de anos chuvosos em relação à média, as exceções foram 2000 (2617,96 mm), 2009
$(2674,30 \mathrm{~mm})$ e $2011(2372,70 \mathrm{~mm})$ - (Figura 3a). As exceções mencionadas anteriormente correspondem aos episódios de La Niña, que por sua vez são responsáveis pelo aumento considerável de chuva no NEB, principalmente na costa (Correia Filho et al., 2019; Da Silva et al., 2020; Costa et al., 2020).

A variabilidade interanual entre anos chuvosos e secos no munícipio de Maceió foram destacados na Figura 3b. No período P1, houve maior ocorrência de anos chuvosos em relação aos anos secos, com destaque para anos de 1964 $(33,58 \%), 1969(45,84 \%), 1977(35,77 \%)$ e 1989 $(62,41 \%)$, isto é, houve aumento considerável da chuva, as exceções foram os anos de 1981 ($29,99 \%)$ e $1983(-34,94 \%)$ com redução de chuva em Maceió. Situação contrária no período P2, onde os anos secos se destacaram, principalmente, os anos de $1993(-54,33 \%), 1998(-40,72 \%)$ e $1999(-31,59 \%)$, as exceções foram os anos de $2000(42,41 \%)$ e $2009(45,48 \%)$, com excesso de chuva em Maceió. Em geral, o período P1 foi período de ocorrências de chuvas superiores à média histórica, enquanto no período P2 houve redução significativa da chuva no munícipio de Maceió. As fases do ENOS são responsáveis diretas pela variabilidade da chuva interanual em Maceió, porém não se descarta a influência do modo de variabilidade climática Oscilação Decadal do Pacífico (ODP), identificado anteriormente por Lyra et al. (2017), visto que a variabilidade decadal está diretamente ligada à mudança de fase da ODP, que por sua vez intensifica as fases do ENOS (Lyra et al., 2017; Costa et al., 2020).

Vale ressaltar que Maceió encontra-se na mesorregião do Leste Alagoano e, tal mesorregião passou por diversas mudanças no uso e ocupação da terra a partir da década de 1990, particularmente a expansão desordenada continua na parte alta de cidade nos dias atuais (Correia Filho et al., 2019) e que resultou na formação e intensificação da Ilha de Calor Urbano (ICU) e, assim em mudanças significativas na dinâmica da chuva na escala decadal (Santos et al., 2021a), sendo confirmado recentemente via Normalized Difference Vegetation Index (NDVI) por Santos et al. (2021b) e também em relação aos cenários futuros de precipitação em Maceió, definidos pela Representative Concentration Pathway (RCP), neste caso RCP 4.5 e RCP 8.5 no estudo realizado por Oliveira et al. (2021). 

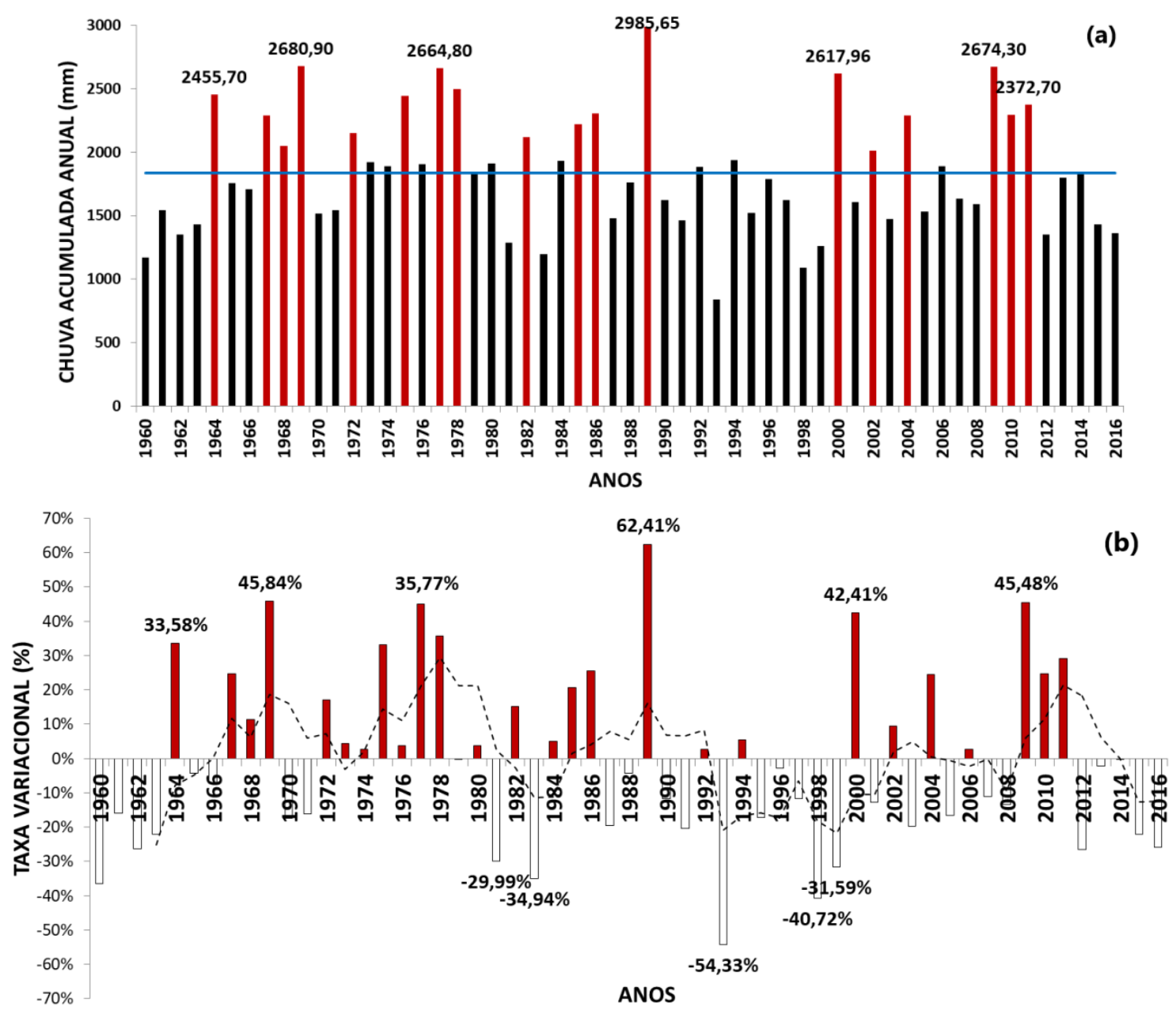

Figura 3. Distribuição temporal da (a) chuva acumulada anual versus a média (mm) e (b) taxa variacional (\%) e média móvel (tracejada) no período de 1960-2016 para o município de Maceió.

\section{Variabilidade Mensal da Chuva}

A Figura 4 mostra a distribuição mensal da chuva e suas variações mensais no munícipio de Maceió. A sazonalidade das chuvas em Maceió tem três estações do ano bem definidas, similar aos resultados obtidos para o Estado de Alagoas via boxplot (Costa et al., 2020) - (Figura 4a). A estação chuvosa (ou quadratura chuvosa), correspondente a abril a julho e foi responsável por $59,52 \%$ da chuva, seguido da estação seca (outubro a fevereiro), com $17,20 \%$ da chuva e a estação de transição (março, agosto e setembro) correspondente a $23,28 \%$ da chuva no período de estudo. A sazonalidade da chuva em Maceió se deve aos seguintes sistemas meteorológicos responsáveis são: circulações de brisas; Vórtices Ciclônicos em Altos Níveis (VCAN); Alta Subtropical do Atlântico Sul (ASAS), Distúrbio Ondulatório de Leste (DOL) e Perturbação Ondulatória dos Alísios (POA) e as circulações de brisas marítima/terrestre e lagunar (Lyra et al.,
2017; Costa et al., 2020; Da Silva et al., 2020; Souza et al., 2021; Oliveira Júnior et al., 2021). Comparativamente a taxa variacional entre os dados observados versus as normais climatológicas apontaram uma diminuição significativa entre a chuva observada em relação a normal 1 (1961-1990), principalmente na estação chuvosa, as exceções foram os meses de agosto (5\% de aumento) e novembro ( $40 \%$ de aumento). Situação contrária, ocorreu entre a chuva observada em relação a normal 2 (1981-2010), destaque para os meses de fevereiro, março e abril (entre 10 a $20 \%$ de aumento), outubro e dezembro (entre 5 a $15 \%$ de aumento) - (Figura 4b). Os resultados obtidos corroboram com os resultados obtidos por Oliveira et al. (2021), em relação aos cenários RCP 4.5 e RCP 8.5 de precipitação em Maceió.

As variações na chuva mensal se devem ao aumento no grau de urbanização nas últimas décadas que ocorreu no munícipio de Maceió e, 
principalmente na parte alta (Nascimento et al., 2018; Correia Filho et al., 2019). Não se descarta que tal aumento esteja ligado às mudanças de uso e ocupação da terra e o processo de urbanização no Leste Alagoano, e assim aumentaram de forma significativa as chuvas convectivas (Costa et al., 2020; Santos et al., 2021a; Santos et al., 2021b). Os meses identificados anteriormente com aumento e diminuição da chuva mensal corroboram com o estudo realizado recentemente por Maia et al. (2020) de armazenamento e reuso da água e os benefícios financeiros, ambientais e a preservação dos recursos hídricos. Vale destacar

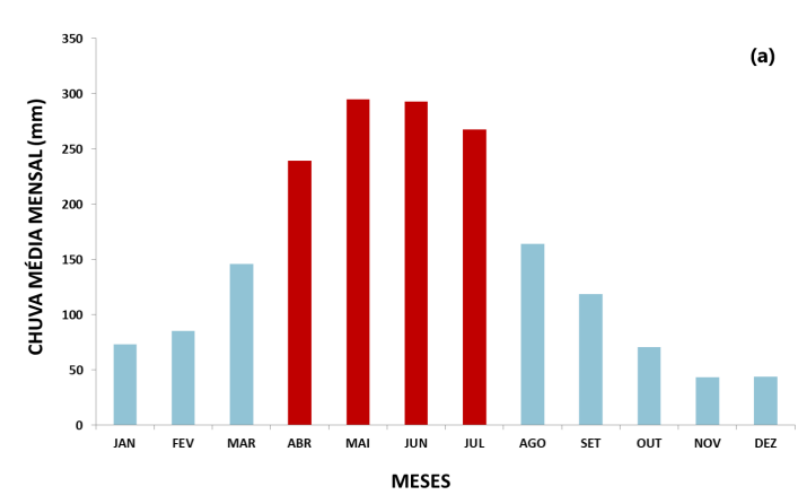

que o aumento das chuvas pode aumentar as condições de vulnerabilidade socioambiental no município de Maceió (IBGE, 2020; Oliveira Júnior et al., 2021), principalmente nas favelas que estão situadas nas encostas, depressões (grotas) ou áreas sob influência do regime de cheias dos rios e lagunas (Nascimento et al., 2018). Já a diminuição das chuvas pode ocasionar o aumento de incêndios urbanos e no entorno do município de Maceió, identificados recentemente por Oliveira Júnior et al. (2020) e Santos et al. (2021a).

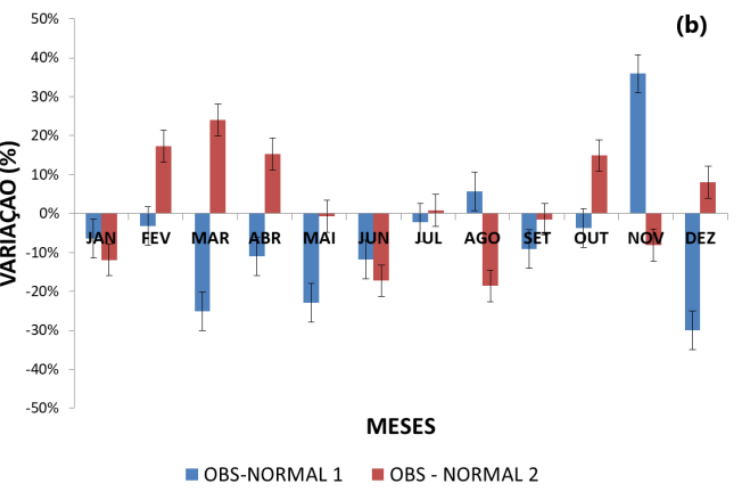

Figura 4. Chuva média mensal (mm) - (a) e (b) taxa variacional (\%) entre dados observados e as normais climatológicas (normal 1 = 1961-1990 e normal 2 = 1981-2010), com respectivos erros no período de 1960-2016 para o município de Maceió.

A Figura 5 mostra o gráfico de dispersão dos dados observados versus produto CHELSA apresentou maior dispersão em relação à linha 1:1 no munícipio de Maceió, a partir de 300 mm.mês1.

Quanto menor o desvio de linha de 1:1, maior a concordância entre os dados observados e estimados, sendo testados para outros produtos de precipitação (CHIRPS - Climate Hazards Group InfraRed Precipitation with Station e PERSIANNCDR - Precipitation Estimation from Remotely Sensed Information using Artificial Neural
Networks - Climate Data Record) no Brasil (Costa et al., 2019; Sobral et al., 2020). O coeficiente $R^{2}$ foi de $80 \%$ e os erros obtidos ( $E P E, 6,58 \mathrm{~mm}$ e RMSE, $18,76 \mathrm{~mm}$ ) foram de acordo com os resultados obtidos em estudos realizados anteriormente em nível de estado (Sobral et al., 2020), regional (Paredes-Trejo et al., 2017) e país (Costa et al., 2019). O coeficiente $r$ foi de 0,89 , com a precisão muito alta, similar aos resultados obtidos recentemente por Oliveira Júnior et al. (2021) para a Região Metropolitana de Maceió (RMM). 


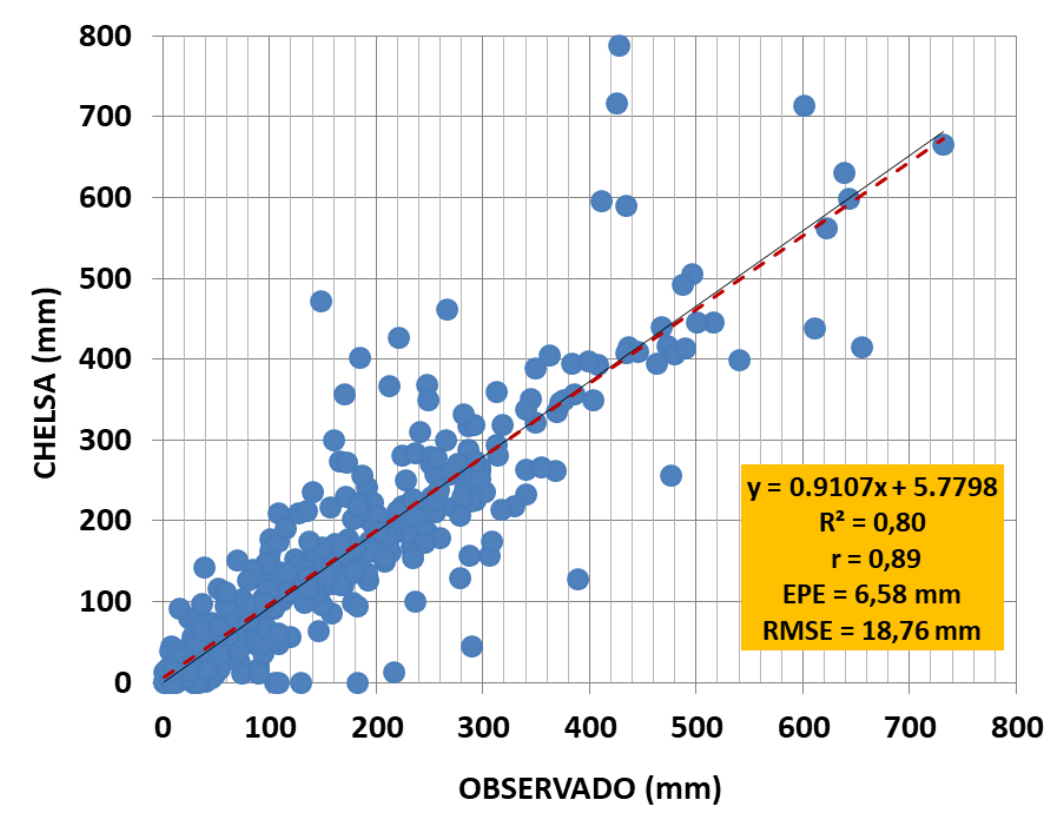

Figura 5. Diagrama de espalhamento entre dados observados e o produto CHELSA (mm) no período de 1979-2013 para o município de Maceió.

Após a validação do produto CHELSA via indicadores estatísticos, na Figura 6 mostra a distribuição espacial da chuva mensal via produto CHELSA para o munícipio de Maceió. A estação chuvosa (ou quadratura chuvosa), correspondente aos meses de abril a julho mostrou a formação e um gradiente pluviométrico bem definido entre a costa e parte alta do município de Maceió. A topografia (Figura 1) influencia o regime de chuva na estação chuvosa e destaque para as regiões administrativas (parte da R4, R5 e R6 - Figura 2) com maiores registros de chuva, superior a 315 mm.mês ${ }^{-1}$.

O munícipio de Maceió é pertencente à mesorregião do Leste Alagoano e, portanto, é influenciada diretamente por diversos sistemas meteorológicos que atuam na costa, principalmente as POA, ASAS, VCAN, circulações de brisas e os DOL - (Costa et al., 2020; Da Silva et al., 2020; Souza et al., 2021). A estação seca e de transição apresentaram uma mudança gradual da chuva no município de Maceió, destaque para a diminuição considerável da chuva na costa, inferior a $105 \mathrm{~mm} \cdot \mathrm{mês}^{-1}$. Vale ressaltar que as regiões destacadas anteriormente (parte da R4, R5 e R6) na estação chuvosa, com maiores registros de chuva ainda se destacam em ambas as estações seca e de transição.

Júnior., J., F., O., Souza., P., H., A., Souza., E., O., Vanderlei., M., H., G., S., Filho., W., L., F., C., Santos., C., T., B., Batista., B., A., Santiago., D., B., Gois., G. 


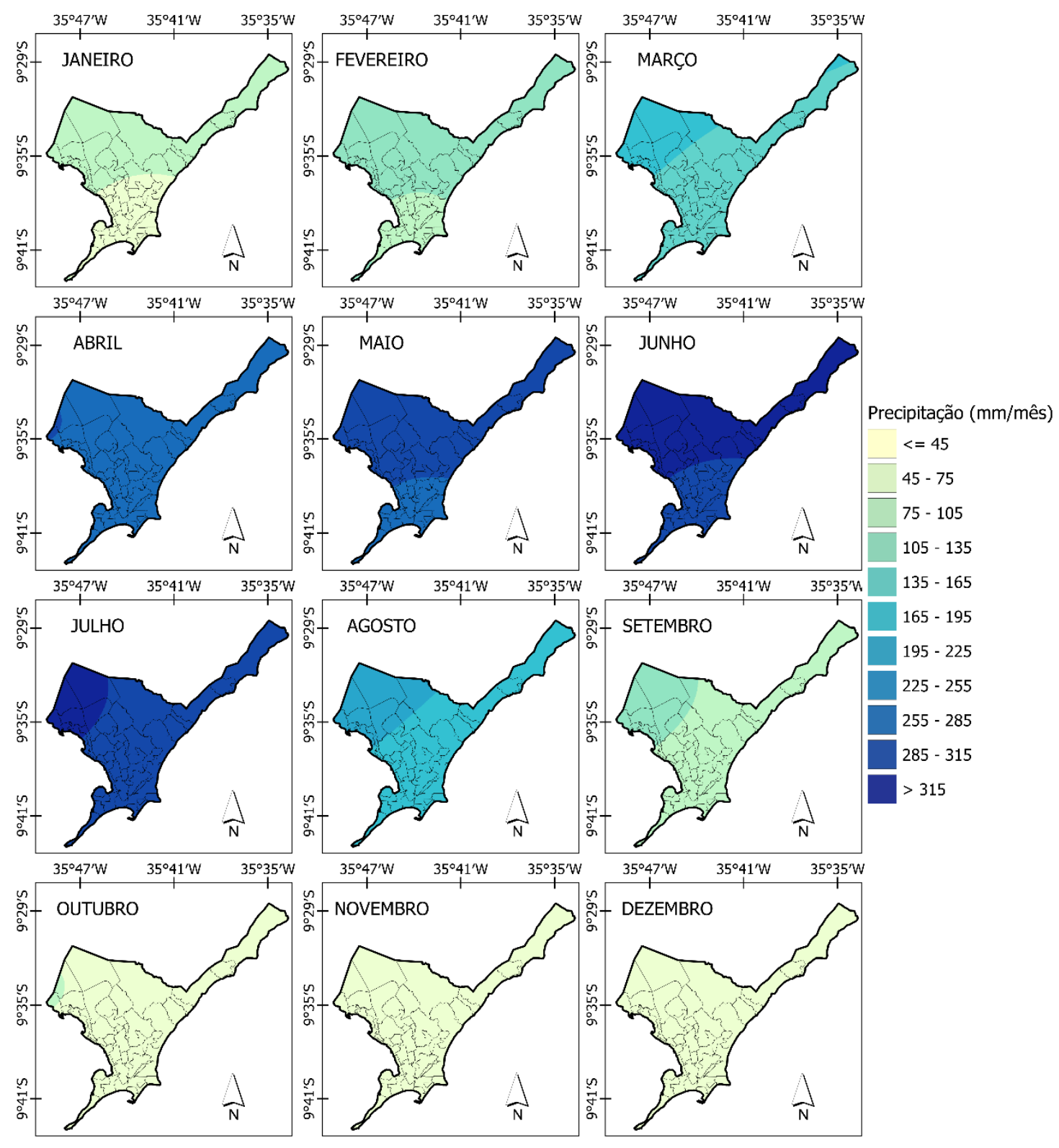

Figura 6. Distribuição espacial da chuva mensal via produto CHELSA (mm) no período de 1979 a 2013 para o município de Maceió.

\section{Conclusões}

A chuva anual no município de Maceió possui dois períodos bem definidos: entre 1960 a 1990 (P1) e entre 1990 a 2016 (P2) na série temporal. Ambos os períodos temporais alternam em anos chuvosos e secos, sendo que tal variabilidade temporal é diretamente ligada à atuação do ENOS, com destaque para as fases de La Niña e Neutro (anos chuvosos) e El Niño (anos secos).

$\mathrm{Na}$ chuva mensal, há aumento significativo nos meses chuvosos em Maceió, na série temporal avaliada. Não se descarta a influência do processo de urbanização e as alterações no uso e ocupação da terra, particularmente a partir da década de 1990, sendo necessária uma investigação sobre uso e ocupação versus chuva em Maceió.

A distribuição espacial da chuva mensal em Maceió via produto CHELSA mostra a formação de um gradiente pluviométrico entre a região da costa e parte alta do município. A variação da topografia influencia o regime de chuva, principalmente os bairros pertencentes a R4, R5 e R6 com maiores registros de chuva, sendo os bairros mais populosos. O munícipio de Maceió é próxima costa e, portanto, é influenciado por diversos sistemas 
meteorológicos, principalmente as circulações das brisas, VCAN, ASAS, POA e os DOL's.

Os dados do CHELSA são satisfatórios a partir dos indicadores estatísticos e, portanto podem ser usados na avaliação espaço-temporal das chuvas e nos estudos de tendências e de eventos extremos no município de Maceió.

Os autores sugerem a implantação de uma rede telepluviométrica nas regiões administrativas do município de Maceió e, assim monitorar a chuva em tempo real similar ao Sistema AlertaRJ.

\section{Referências}

Armstrong, M.S., Kiem, A.S., Vence, T.R., 2020. Comparing instrumental, palaeoclimate, and projected rainfall data: Implications for water resources management and hydrological modelling. Journal of Hydrology: Regional Studies 31, 100728. https://doi.org/10.1016/j.ejrh.2020.100728

Baklanov, A., Molina, L.T., Gauss, M., 2016. Megacities, air quality and climate. Atmospheric Environment, 126, 235-249. https://doi.org/10.1016/j.atmosenv.2015.11.05 9

Boretti, A., Rosa, L., 2019. Reassessing the projections of the World Water Development Report. NPJ Clean Water 2, 1-15. https://doi.org/10.1038/s41545-019-0039-9

Correia Filho, W.L.F., Santiago, D.B., Oliveira Júnior, J.F., Silva Junior, C.A., 2019a. Impact of Urban Decadal Advance on Land Use and Land Cover and Surface Temperature in the City of Maceió, Brazil. Land Use Policy 87, 104026.

https://doi.org/10.1016/j.landusepol.2019.1040 26

Correia Filho, W.L.F., Oliveira Júnior, J.F., Santiago, D.B., Terassi, P.M.B., Teodoro, P.E., Gois, G., Blanco, C.J.C., Souza, P.H.A., Costa, M., Santos, P.J., 2019b. Rainfall variability in the Brazilian northeast biomes and their interactions with meteorological systems and ENSO via CHELSA product. Big Earth Data 3 ,

315-337. https://doi.org/10.1080/20964471.2019.169229 8

Costa, J., Pereira, G., Siqueira, M.E., Cardozo, F., Silva, V.V., 2019. Validação dos Dados de Precipitação Estimados pelo CHIRPS para o Brasil. Revista Brasileira de Climatologia 24, 228-243.

http://dx.doi.org/10.5380/abclima.v24i0.60237
Costa, M.S., Oliveira Júnior, J.F., Santos, P.J., Correia Filho, W.L.F., Gois, G., Blanco, C.J.C., Teodoro, P.E., Silva Junior, C.A., Santiago, D.B.; Souza, E.O, Ferraz Jardim, A.M.R., 2020. Rainfall extremes and drought in Northeast Brazil and its relationship with El Niño-Southern Oscillation. International Journal of Climatology, 41, 2111-2135. https://doi.org/10.1002/joc.6835

Da Silva, D.F., Lima, M.J.S, Souza Neto, P.F, Gomes, H.B, Silva, F.D.S, Almeida, H.R.R.C, Pereira, M.P.S, Costa, R.L., 2020. Caracterização de eventos extremos e de suas causas climáticas com base no Índice Padronizado de Precipitação Para o Leste do Nordeste. Revista Brasileira de Geografia Física 13, 449-464. https://doi.org/10.26848/rbgf.v13.2.p449-464

FAO - Food and Agriculture Organization. State of Food and Agriculture - Climate Change, Agriculture, and Food Security. Rome. 2016.

IBGE - Instituto Brasileiro de Geografia e Estatística. Área da unidade territorial: Área territorial brasileira. Rio de Janeiro, 2020. Disponível em: cidades. ibge.gov.br/brasil/al/panorama. Acesso: 24 mai.2021.

IPCC - Intergovernmental Panel On Climate Change - Global Warming of $1.5^{\circ} \mathrm{C}$, An Impacts of Global Warming of $1.5^{\circ} \mathrm{C}$ Above Pre-Industrial Levels and Related Global Greenhouse Gas Emission Pathways, in the Context of Strengthening the Global Reponse to the Threat of Climate Change, Sustainable Development, and Efforts to Eradicate Poverty. IPCC Special Report, 2018.

Karger, D.N., Conrad, O., Böhner, J., Kawohl, T., Kreft, H., Soria-Auza, R. W., Zimmermann, N.E., Linder, H. P., Kessler, M., 2017. Climatologies at high resolution for the earth's land surface areas. Scientific Data 4, 170122.https://doi.org/10.1038/sdata.2017.122

Lima, A.O., Lyra, G.B., Abreu, M.C., OliveiraJúnior, J.F., Zeri, M., Cunha-Zeri, G., 2021. Extreme rainfall events over Rio de Janeiro State, Brazil: Characterization using probability distribution functions and clustering analysis. Atmospheric Research 247, 105221.

https://doi.org/10.1016/j.atmosres.2020.10522 1

Lyra, G.B., Oliveira-Júnior, J.F., Gois, G., CunhaZeri, G., Zeri, M., 2017. Rainfall variability over Alagoas under the influences of SST anomalies. Meteorology and Atmospheric 
Physics

129 ,

157-171.

https://doi.org/10.1007/s00703-016-0461-1

Maia, A.C.J., Dâmaso, M.L.F., Silva, S.G., Silva Junior, T.L., Melo, M.B., 2020. Reservatório para Armazenamento de Água da Chuva em Edificações na Cidade de Maceió-AL. Cadernos de Graduação - Ciências Exatas e Tecnológicas 6, 219-231. Disponível: https://periodicos.set.edu.br/fitsexatas/article/vi ew/9205. Acesso: 24 mai.2021.

Marengo, J.A., Alves, L.M., Alvala, R.C.C.; Cunha, A.P.M.A., Brito, S.B.; Moraes, O.L.L., 2018. Climatic characteristics of the 20102016 drought in the semiarid Northeast Brazil region. Anais da Academia Brasileira de Ciências $\quad 90, \quad 1973-1985$. https://doi.org/10.1590/00013765201720170206

Nascimento, M.C., Lombardo, M.A., Guimarães Junior, S.A.M., Andrade, E.L., 2018. Análise da Vulnerabilidade Físico-Ambiental Causada Pelas Chuvas Intensas na Região Metropolitana de Maceió. Caminhos da Geografia (UFU. Online) 19, 268288.https://doi.org/10.14393/Hygeia196718

NOAA/CPC - National Oceanic and Atmospheric Administration/Climate Prediction Center. Disponível em: http://www.cpc.ncep.noaa.gov/products/analys is_monitoring/ensostuff/ensoyears.shtml.

Acesso em 20/01/2021.

Oliveira Júnior, J.F., Gois, G., SILVA, E. B., Teodoro, P.E., Johann, J., Silva Junior, C.A., 2019. Non-parametric tests, multivariate analysis and descriptive and exploratory statistics applied to reported dengue cases in Brazil. Environmental Monitoring and Assessment 191, 473-491. https://doi.org/10.1007/s10661-019-7583-0

Oliveira, L.P.M., Santos Silva, F.D., Costa, R. L., Rocha Júnior, R.L., Gomes, H.B., Pereira, M.P.S., Monteiro, L.A., Silva, V.P.R., 2021. Impacto das Mudanças Climáticas na Produtividade da Cana de Açúcar em Maceió. Revista Brasileira de Meteorologia 35, 969980. https://doi.org/10.1590/010277863550107

Oliveira-Júnior, J.F., Correia Filho, W.L.F., Alves, L.E.R., Lyra, G.B., Gois, G., Silva Junior, C.A., Santos, P.J., Sobral, B.S., 2020. Fire Foci Dynamics and their relationship with socioenvironmental factors and meteorological systems in the state of Alagoas, Northeast Brazil. Environmental Monitoring and
Assessment 192, 654. https://doi.org/10.1007/s10661-020-08588-5

Oliveira-Júnior, J.F., Correia Filho, W.L.F., Santiago, D.B., Gois, G., Costa, M., Silva Junior, C.A., Teodoro, P.E., Freire, F.M., 2021. Rainfall in Brazilian Northeast via in situ data and CHELSA product: mapping, trends, and socio-environmental implications. Environmental Monitoring and Assessment 193, 263-281. https://doi.org/10.1007/s10661021-09043-9

Paredes-Trejo, F.J., Barbosa, H.A., Lakshmikumar, T.V., 2017. Validating CHIRPS-based satellite precipitation estimates in Northeast Brazil. Journal of Arid Environment 139, 26-40. https://doi.org/10.1016/j.jaridenv.2016.12.009

Plano Municipal de Assistência Social de Maceió (PMAS-MACEIÓ) 2014 - 2017. Plano Diretor da Cidade de Maceió, 106 p., 2014.

Santos, E.M.S., Correia Filho, W.L.F., Gomes, H. B., Oliveira Júnior, J. F., Falcão, N.A.M., Costa, M., Gois, G., 2021a. EspaçoTemporalidade dos Focos de Calor na Região Metropolitana de Maceió. Revista Brasileira de Meteorologia 35, 1029-1043. https://doi.org/10.1590/0102-7786355000091

Santos, F.S., Gomes, H.B., Barros, G.V.P., Alves, L.E.R., Silva, D.F., Costa, R.L., Santos Silva, F.D., Oliveira Júnior, J.F., 2021b. Análise Sazonal dos Parâmetros Biofísicos Utilizando o Sensor MODIS para o Estado de Alagoas. Revista Brasileira de Meteorologia 35, 955 968. 77863550093

Santos, S.R.Q., Cunha, A.P.M.A., Ribeiro-Neto, G.G., 2019. Avaliação de dados de precipitação para o monitoramento do padrão espaço-temporal da seca no nordeste do Brasil. Revista Brasileira de Climatologia 25, 80-100. http://dx.doi.org/10.5380/abclima.v25i0.62018

Sobral, B.S., Oliveira-Júnior, J.F., Alecrim, F.B., Gois, G., Muniz Junior, J.G.R., Terassi, P. M.B., Pereira Junior, E.R., Lyra, G.B., Zeri, M., 2020. PERSIANN-CDR based characterization and trend analysis of annual rainfall in Rio de Janeiro State, Brazil. Atmospheric Research 238, 104873.https://doi.org/10.1016/j.atmosres.202 0.104873

Souza, E.O., Costa, M.S., Oliveira Júnior, J.F., Gois, G., Mariano, G.L., Costa, C.E.S., Correia Filho, W.L.F., Santiago, D.B., 2021. Estimativa e Espacialização da Erosividade em Mesorregiões Climáticas no Estado de 
Alagoas. Revista Brasileira de Meteorologia 35, 769-783. https://doi.org/10.1590/010277863550005

Terassi, P.M.B., Oliveira Júnior, J.F., Gois, G., Oscar Júnior, A.C., SOBRAL, B.S., Biffi, V.H.R.; Blanco, C.J.C., Correia Filho, W.L.F.,
Vijith, H., 2020. Rainfall and erosivity in the municipality of Rio de Janeiro - Brazil. Urban Climate 33,100637 . https://doi.org/10.1016/j.uclim.2020.100637 